\title{
Patient Consultation in the Department of Immunology and Medical Zoology at St. Marianna University School of Medicine between 2001-2019 with an Interest in Parasitic Diseases in Japan
}

\author{
Jun Shimizu, Kenji Takai, and Noboru Suzuki
}

(Received for Publication: February 15, 2021)

\begin{abstract}
Objective We aimed to summarise recent epidemiological findings regarding parasitic diseases and other animal-derived diseases based on our studies in Kawasaki City with a reference to other areas in Japan.

Methods We conducted a retrospective survey of animal-derived diseases reported between 2001-2019 in the Department of Immunology and Medical Zoology of St. Marianna University School of Medicine.

Results Thirty-two patients were referred to our department from neighboring hospitals for evaluation and consultation regarding suspicious parasitic diseases and other animal-derived diseases. Twenty patients were diagnosed as having parasitic disease or some other animal-derived disease. Twelve patients remained undiagnosed despite detailed examination of their clinical samples. Among the 20 patients with parasitic disease, six and four patients were diagnosed with diphyllobothriasis and anisakiasis, respectively. Tick bites without obvious microbial infection were found in two cases.

Conclusion We found that parasitic diseases reported over the past two decades were mostly of helminthic origins and food-borne. Although the number of cases was small, we identified two cases of tick bites in Kawasaki City. Therefore, careful attention should be paid to the emergence of severe tick-borne diseases even in urban areas.
\end{abstract}

\section{Key words}

Parasitic diseases, tick bites, diphyllobothriasis, anisakiasis, tick-borne diseases

\section{Introduction}

When making a diagnosis of parasitic disease or other animal-derived disease, special experience/ technique is often required because of their rarity. Since its establishment, our laboratory has been accepting consultations on parasitic diseases from hospitals around St. Marianna University Hospital in Kawasaki City.

We previously reported the epidemiological findings of cases sent for consultation between 1993$2000^{11}$. For the diagnosis of parasitic diseases and other animal-derived diseases, the main approach includes morphological characterization of the specimen collected by the physician treating the affected patient.

Clinical sample collection and timing are important for the precise diagnosis of parasitoses and arthropod bites; such clinical characteristics are observed in cases of paragonimiasis ${ }^{2}$, anisakiasis ${ }^{3)}$, spiruroid nematodiasis ${ }^{4)}$, echinococcosis ${ }^{5)}$, and Japanese spotted fever ${ }^{6}$. Japanese spotted fever is known to be a tick-borne disease, where the tick acts as the vector of a microbial disease.

Our previous publication disclosed that we consulted on 32 cases between 1993-2000, and 10 cases were diagnosed morphologically as having a parasitic disease $^{1)}$. Among these, diphyllobothriasis was observed in four cases and anisakiasis was observed in three cases. Tick bites were observed in four cases. 
In this study, we summarised the consultation data from the Department of Immunology and Medical Zoology, St. Marianna University School of Medicine between 2001-2019. We estimated the local incidence of parasitic diseases and other animalderived diseases observed in Kawasaki City. The incidence of parasitic diseases was reported to be rare and lower than the cases reported in our previous study $^{1)}$. Nonetheless, physicians should remember that patients can be infected with parasites and suffer from arthropod bites even in urban areas in Japan.

This study was approved by the institutional review board of St. Marianna University School of Medicine (No. 4931). We conducted our research according to the principles expressed in the Declaration of Helsinki.

\section{Materials and Methods}

\section{Patients}

Thirty-two patients were referred to the Department of Immunology and Medical Zoology between 2001-2019 (Table 1). Among these patients, 20 were diagnosed as having parasitic diseases or some other animal-derived diseases. We obtained the relevant specimens for 28 cases directly from physicians treating the patients. The remaining four patients were interviewed by the consulting physicians from our department. We examined the specimens morphologically, serologically, and using DNA sequence technology. Twenty-eight patients were referred from St. Marianna University Hospital, and four patients from other nearby hospitals.

\section{Examination of parasite and bug specimens}

We employed the formalin-ether centrifugation method for parasite-containing stools ${ }^{7}$. Diphyllobothrium was identified based on adult morphology and the morphology of mature eggs retrieved from adult uterus. Anisakis specimens were identified based on their morphology.

The Diphyllobothrium specimen in one case was identified using DNA diagnosis; the presence of the species specific cyclooxygenase 1 gene in the mitochondrial $\mathrm{DNA}^{8)}$ was analysed using polymerase chain reaction. Anisakis was identified in one case using DNA analysis; the base sequences of the internal transcribed spacer 2 (ITS2) region of ribosomal DNA were examined ${ }^{9)}$.

\section{Examination of Giemsa-stained blood smear specimen}

This study included one suspected case of malaria. In the suspected case, the blood smear was examined microscopically ${ }^{10)}$.

\section{Examination of serum specimen}

This study included one patient with cervical lymphadenitis. The serum specimen was serologically examined for Paragonimus westermani, Paragonimus miyazakii, Fasciola hepatica, Clonorchis sinensis, Sparganum mansoni, Cysticercus cellulosae, Dirofilaria immitis, Toxocara canis, Ascaris lumbricoides suum, Anisakis simplex, Gnathostoma, and Strongyloides stercoralis ${ }^{11}$.

\section{Results}

We found that the most frequent parasitosis among cases that required our consultation was diphyllobothriasis due to Diphyllobothrium nihonkaiense, which was noted in six patients, followed by anisakiasis due to Anisakis spp. in four patients. They were all diagnosed by morphological examination, followed by an additional DNA identification based confirmation in one patient with diphyllobothriasis and in one patient with anisakiasis. Regarding symptoms, one patient with diphyllobothriasis had diarrhea, two patients were asymptomatic, and related symptoms were unclear in three patients.

Among four patients with anisakiasis, one patient coughed up the larva while vomiting. One patient observed the larva in the stool; in the other two patients, the larvae were detected incidentally by physicians on endoscopic examination.

In one patient with enterobiasis and in one patient with ascariasis, the etiologic agents were identified morphologically. In a patient with an abnormal subcutaneous mass, histological examination of the biopsy specimen identified sparganosis.

One patient with cervical lymphadenitis had a higher titre of anti-toxoplasma IgG without any detectable anti-toxoplasma IgM titre. After careful examination, a diagnosis of toxoplasmosis was made.

Two cases of tick bites, in which the ticks were pulled out by the patients themselves, demonstrated contrasting clinical courses. One patient visited the hospital at night on the same day of tick-bite; the tick-bite occurred on a camp site in a different prefecture, approximately $100 \mathrm{~km}$ northeast of Kawasaki City. He removed the tick himself. He experienced no redness, pain, or pruritus. The other patient noticed 
the tick 2 days after the bite in a mountainous area of a different prefecture, approximately $200 \mathrm{~km}$ northwest of Kawasaki City. He removed the tick by pulling it out. He visited a nearby hospital two days later. The mouth parts, of the tick remained in the skin. He displayed redness in the stratum corneum and developed granuloma; therefore, the physician removed the mouth parts by surgical excision. The etiologic ticks were Ixodes spp. in the former and Ixodes persulcatus in the latter.

\section{Discussion}

We summarised the records of patients with parasitic diseases and other animal-derived diseases consulted at our Department between 2001-2019 (Table 1). Our former study ${ }^{1)}$ included 21 patients, giving an average of 2.6 patients per year. This study reported 20 patients over the last 19 years, giving an average of 1.0 patient per year. The decline in the number of patients referred in the most recent period may reflect improvements in hygienic and environmental situation in Kawasaki City. The improvement in hygienic status was partly supported by the fact that the Ordinance for Enforcement of School Health and Safety Act of Japan was revised and parasite egg examination of pupils has not been required since $2016^{12)}$.

The most frequent patients referred to us between 1993-2000 were with cases of diphyllobothriasis and tick bites, followed by anisakiasis ${ }^{1)}$. We found that diphyllobothriasis was most frequent, followed by anisakiasis and tick bites in the present study.

Matsuoka et al. reported that the most frequent cases were of ascariasis (14 cases), followed by malaria (nine cases) and diphyllobothriasis (nine cases) ${ }^{13)}$ in the last 5 years of the 20th century, in the Japanese countryside. Another study showed that, in the last decade of the 20th century, the most frequent cases were of diphyllobothriasis (46 cases), followed by ascariasis ( 31 cases) and anisakiasis ( 30 cases $)^{14)}$. The difference in disease prevalence may reflect the difference between the times and regions of the above-mentioned three studies. Indeed, several recent studies in Japan ${ }^{15,16)}$ demonstrated similar results to those of our studies; particularly, in terms of disease incidence.

Thus, diphyllobothriasis has been prevalent in Japan for the past 5 decades ${ }^{17}$. Global food delivery systems have made this disease prevalent worldwide $^{18)}$. Anisakiasis is also frequently seen in Ja$\operatorname{pan}^{19)}$. As mentioned, some patients with diphyllobo- thriasis and those with anisakiasis were asymptomatic. Therefore, we should be aware of the possibility of diphyllobothriasis and anisakiasis even if the patient lacks any symptoms but consumed raw fish.

There was one case each of giardiasis, toxoplasmosis, blastocystosis, ascariasis, enterobiasis, sparganosis, and cysticercosis. We noted characteristic subtle and severe symptoms in some patients, including diarrhea in the patient with giardiasis. Furthermore, subcutaneous abscess in the patient with sparganosis, and pyelitis and intracranial calcification in the patient with cysticercosis were also included.

Two tick-bite cases lacked evidence of microbial infection, such as severe fever with thrombocytopenia syndrome, Japanese spotted fever, or Lyme disease. Nevertheless, tick-borne diseases are likely to be encountered even in urban areas near Tokyo.

In this study we report that parasitoses and tick bites appear to occur rather consistently among residents of Kawasaki City, taking their leisure/business activity range into consideration. The vast majority of parasitoses reported in this study are not included in the Infectious Diseases Control Law in Japan. As more cases of parasitoses are left unreported to government offices ${ }^{17,19)}$, their reported incidence declines. Their rarity, when they emerge, may make their diagnosis by physicians more difficult. Indeed, the physicians in our studies who suspected parasitosis consulted us as clinical support for their diagnosis may help them to provide appropriate treatments.

Medical school curriculum has become compressed in Japan; therefore, it is difficult for students to be educated sufficiently, especially on parasitic diseases. For making a prompt/proper diagnosis of parasitosis, databases of patient diseases with their occurrence localities, prevalence, major symptoms, and important clinical characteristics may prove to be helpful. Thus, the development of such databases is necessary.

\section{Conflicts of Interest}

The authors have nothing to disclose.

\section{Acknowledgements}

We thank Professor Hiroshi Yamazaki of National Institute of Infectious Diseases and Professor Nobuaki Akao of Tokyo Medical and Dental University, for identification of parasite specimens, and Department of Parasitology, Faculty of Medicine, University of Miyazaki, for serological tests of the serum. 

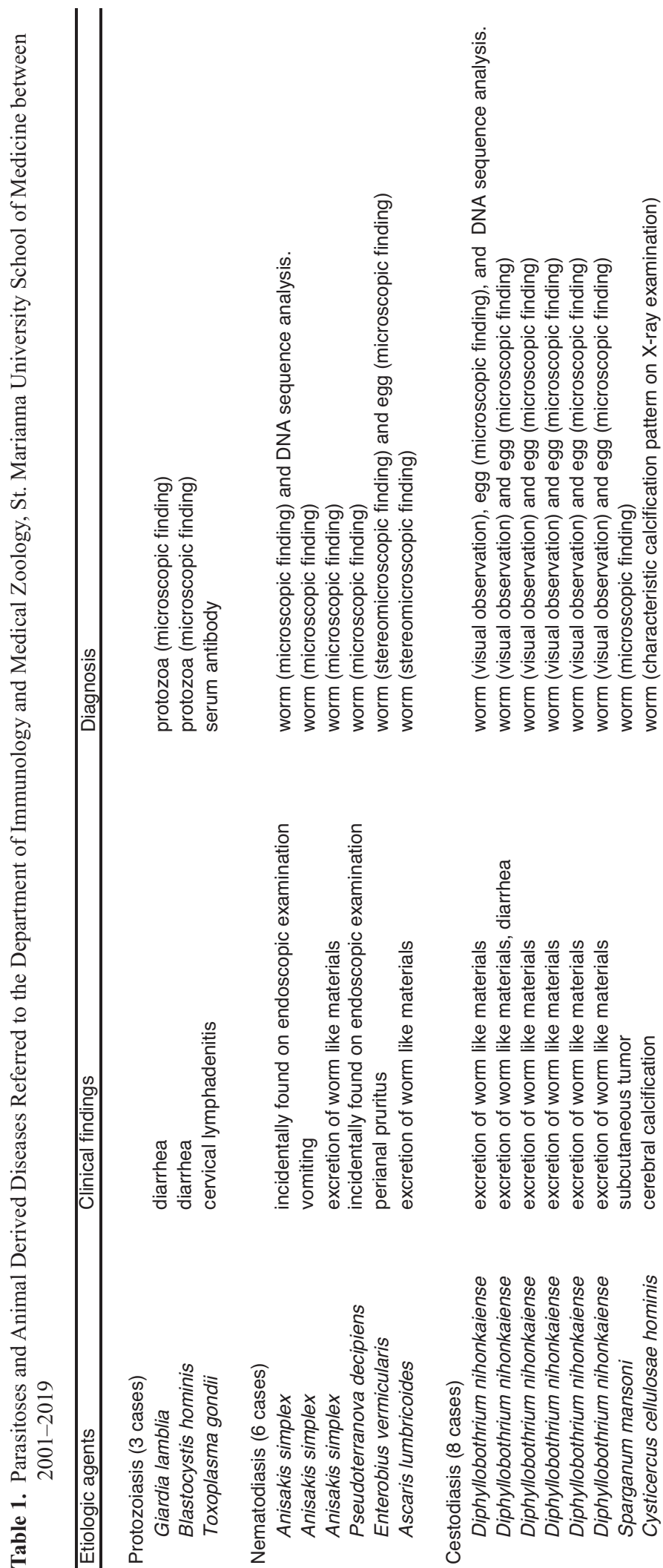

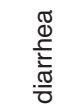

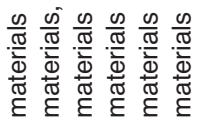

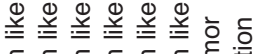

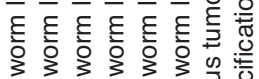



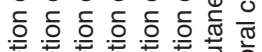

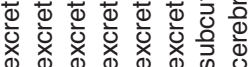

ఖ

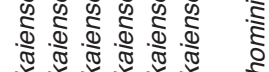

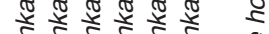

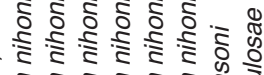
ब.

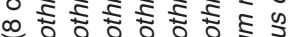

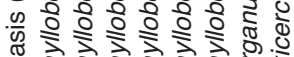
के
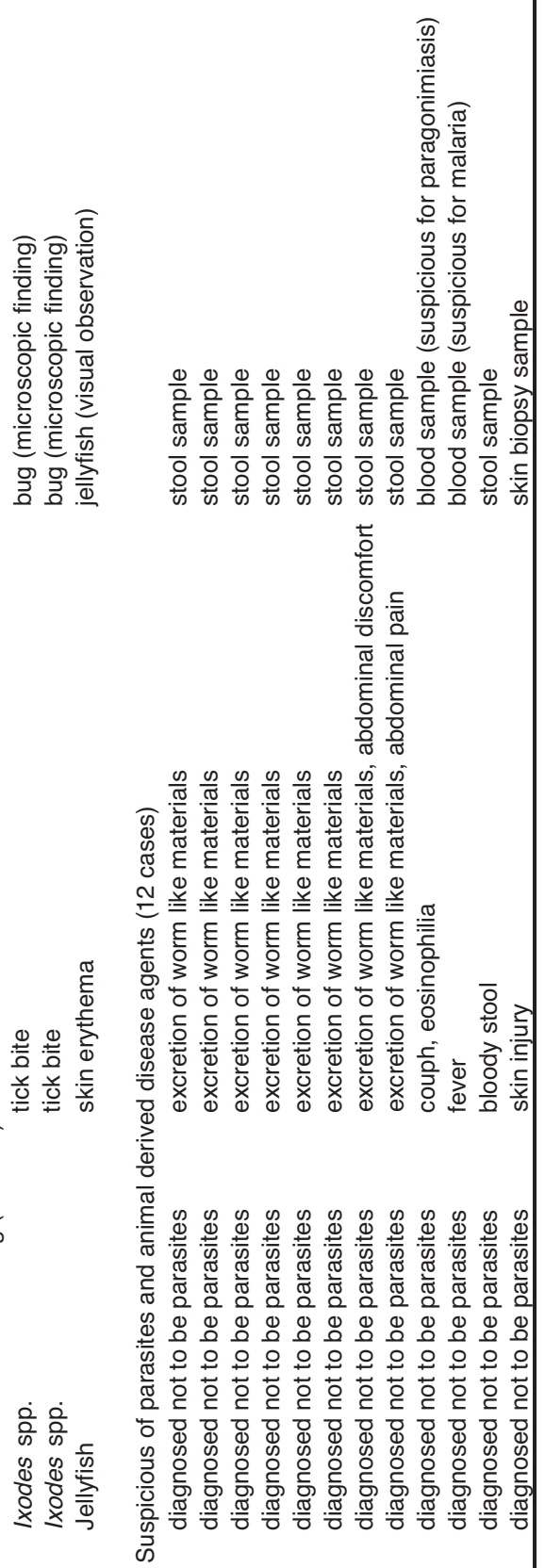


\section{References}

1) Takai K, Ogawa K, Takeno M, et al. Analysis of parasitic diseases consulted with the Department of Immunology and Medical Zoology, St. Marianna University School of Medicine, during the period 1993 to 2000. J St Marianna Univ [In Japanese, abstract in English. This literature was retrieved from JGlobal service: https://jglo bal.jst.go.jp/en/detail?JGLO

BAL_ID=200902129100475647. This literature was also available through the University interlibrary loan system.] 2001; 29: 15-20.

2) Sugiyama H, Shibata K, Kawakami $Y$, et al. Paragonimiasis due to the consumption of wild boar meat in Japan: Contamination levels of lung fluke larvae in muscle samples of wild boars caught in Kagoshima Prefecture. Jpn J Infect Dis 2015; 68: 536-537.

3) Furuya $K$, Nakajima H, Sasaki $Y$, et al. Anisakiasis: The risks of seafood consumption. Niger J Clin Pract 2018; 21: 1492-1494.

4) Kawanaka M, Sugiyama H. Spriruroid nematode. Infect Dis Wkly Rep Jpn (IDWR) [In Japanese] 2001; 3-14: 8-10.

5) National Institute of Infectious Diseases. Echinococcosis, 1999-2018. Byogen Biseibutsu Kenshutsu Joho Geppo [In Japanese] 2019; 40: 33-34.

6) Noguchi M, Oshita S, Yamazoe N, et al. Important clinical features of Japanese spotted fever. Am J Trop Med Hyg 2018; 99: 466-469.

7) Garcia LS. Diagnostic Medical Parasitology, 5th ed, Asm Press, Washington DC, 2007: 782-830.

8) Arizono N, Shedko M, Yamada M, et al. Mitochondrial DNA divergence in populations of the tapeworm Diphyllobothrium nihonkaiense and its phylogenetic relationship with Diphyllobothrium klebanovskii. Parasitol Int 2008; 57: 212216.

9) Kong Q, Fan L, Zhang J, et al. Molecular identification of Anisakis and Hysterothylacium larvae in marine fishes from the East China Sea and the Pacific coast of central Japan. Int J Food Microbiol 2015; 199: 1-7.

10) Ljungström I, Perlmann $H$, Schlichtherle M, et al. Methods in Malaria Research, 4th Edition, The Malaria Research and Reference Reagent Resource Center, Manassas, 2004: 18-19.
11) Nawa $Y$, Nishimura $K$, Matsumoto H. Practical methods for the detection of parasite-specific $\lg \mathrm{G}$ antibody. Jpn J Med Technol [In Japanese] 2001; 29: 1191-1196.

12) Matsunaga N. Details of revision of health examination for students, etc. J Child Health [In Japanese] 2016; 75: 2-7.

13) Matsuoka H, Yoshida S, Hirai M, et al. Reports of parasitic diseases and entomological cases in the Department of Medical Zoology, Jichi Medical School: accumulated cases from five years. Jpn J Infect Dis 2001; 54: 148-150. https:// www.niid.go.jp/niid/images/JJID/ER-3.pdf.

14) Arizono N, Yamada M, Tegoshi T, et al. Parasitic diseases in Kyoto - A Short history and current situation. J Kyoto Prefect Univ Med [In Japanese, abstract in English] 2010; 119; 593611. http://www.f.kpu-m.ac.jp/k/jkpum/pdf/ 119/119-9/arizono09.pdf.

15) Shimada M, Yamamoto DS, Hayakawa EH, et al. Parasitic diseases and other zoonoses referred to the division of Medical Zoology between 2011 and 2014. Jichi Med Univ J [In Japanese, abstract in English] 2015; 38: 71-77. https:// jichi-ir.repo.nii.ac.jp/?action=pa ges_view_main\&active_action=repository_view_main_item_detail\&item_id=98\&item_no=1\&page_id=13\&blo ck_id=21.

16) Hirai N, Yoshikawa M, Ogawa T, et al. Current perspective on parasitic diseases in Japan from an outpatients clinic at Nara Medical University. Clin Parasitol [In Japanese. This literature was available through the University interlibrary loan system.] 2017; 28: 9-12.

17) Yamazaki H, Morishima Y, Sugiyama H. Current occurrence of cestodiasis in Japan. Byogen Biseibutsu Kenshutsu Joho Geppo [In Japanese] 2017; 38: 74-76.

18) Scholz T, Kucht R. Fish-borne, zoonotic cestodes (Diphyllobothrium and relatives) in cold climates: A never-ending story of neglected and (re)-emergent parasites. Food and Waterborne Parasitology 2016; 4: 23-38.

19) National Institute of Infectious Diseases. Foodborne helminth infections. Byogen Biseibutsu Kenshutsu Joho Geppo [In Japanese] 2017; 38: 69-70. 